\title{
DESEMPEÑO COGNITIVO EN PRUEBAS DE LENGUAJE EN NIÑOS DE 6 A 14 AÑOS ESCOLARIZADOS DE LA CIUDAD DE MEDELLÍN.
}

\author{
COGNITIVE PERFORMANCE ON TESTS OF LANGUAGE IN 6-14-YEAR-OLD \\ SCHOOLED CHILDREN IN THE CITY OF MEDELLIN.
}

\author{
Recibido: febrero de 2013 - Revisado: agosto de 2013 - Aceptado: 30 de octubre de 2013 \\ Por: Maria Cristina Giraldo Garcia ${ }^{1}$, Paola Yulieth Velásquez Correa ${ }^{2}$, \\ Maryoris Elena Zapata Zabala ${ }^{3}$, Elizabeth Hoyos Zuluaga. ${ }^{4}$
}

\section{RESUMEN.}

El presente artículo presenta los resultado de investigación el cual busca describir el desempeño cognitivo de los escolares de 6 a 14 años en pruebas de lenguaje, este estudio se desarrolló con 92 participantes con diferentes estratos socioeconómicos se aplicaron pruebas de Lenguaje: Test de asociación controlada de palabras, Test de vocabulario de Boston, Token test, encontramos que las habilidades y desempeño en pruebas de lenguaje no difieren entre sexo y lateralidad, existen diferencias evidenciables en grupos de edades, con influencia del nivel educacional.

\section{PALABRAS CLAVE.}

Desempeño cognitivo, lenguaje, edad, sexo.

\section{ABSTRACT.}

This paper presents results of research which aimed to describe the cognitive performance on tests of language in 6-14-year-old. In this study, the researchers chose a sample population of 92 participants coming from different socioeconomic strata. That is why tests of language, tests of controlled association, Boston Naming Test, and Token Test were applied.

\section{KEY WORDS AND EXPRESSIONS.}

Cognitive performance; Language; and Age.

\footnotetext{
${ }^{1}$ Psicóloga egresada de la Universidad de San Buenaventura, Medellín- Colombia. Actualmente psicóloga de la clínica Antioquia y vinculada al campo clínico en modalidad independiente. Auxiliar de investigación en pregrado del Grupo de Neuropsicología y Conducta, Universidad de San Buenaventura, Medellín. mariacris9010@hotmail.com.

${ }^{2}$ Psicologa egresada de la Universidad de San Buenaventura, Medellín- Colombia. Actualmente vinculada al campo clínico en modalidad independiente. Auxiliar de investigación en pregrado del Grupo de Neuropsicología y Conducta, Universidad de San Buenaventura, Medellin. paoly_47@hotmail.com.

${ }^{3}$ Psicóloga, Magíster en Neuropsicología - Universidad San Buenaventura de Medellín-Colombia. Docente Investigadora, Grupo de Neuropsicología y Conducta, Universidad San Buenaventura de Medell Estadística e informática- Universidad de Medellín. Docente, Universidad de San Buenaventura. Candidata a Magister en Epidemiología- Universidad del Ces. Correo: Elizabeth.hoyos@usbmed.edu.coín. Doctora en Neuropsicología. Maryoris.zapata@usbmed.edu.co.

${ }^{4}$ Estadística e informática - Universidad de Medellín, Colombia. Docente, Universidad de San Buenaventura. Candidata a Magister en Epidemiologia- Universidad del Ces. Correo: Elizabeth.hoyos@usbmed.edu.co.
} 


\section{Introducción.}

La infancia de un niño constituye una etapa especialmente crítica; en esta se configuran dos procesos superiores centrales, el lenguaje y la cognición; que facilitan los desarrollos motrices, perceptivos, afectivos y sociales; los cuales posibilitan una equilibrada adaptación con el mundo circundante. A partir de estos procesos es que durante el período del desarrollo aumentan los conocimientos y habilidades para percibir, pensar, comprender e interactuar con la realidad (Ibáñez, 2008).

El desarrollo cognitivo debe ser visto como el conjunto de trasformaciones que se producen en las características y capacidades del pensamiento en el transcurso de la vida, especialmente durante el período del desarrollo, y por el cual aumentan los conocimientos y habilidades para percibir, pensar, comprender y manejarse en la realidad (Piaget, 1988).

Desde esta perspectiva, el desarrollo cognitivo es entendido no como una estructura estática, sometida a predisposiciones y determinantes biológicos que limitan la capacidad potencial de los individuos para construir los conocimientos, sino como una estructura que permanentemente se transforma, como resultado de sucesivas reestructuraciones que se producen en las múltiples y variadas interacciones que el sujeto establece con otros sujetos, en contextos socioculturales diversos. (Alvarado, 1997).

La comunicación constituye un proceso central a través del cual se intercambian y construyen significados con otros, a lo largo de todo el ciclo vital. Dentro de este proceso, el lenguaje se convierte en vehículo de pensamientos, sentimientos e información, con ello, que la adquisición del lenguaje es necesaria para el bienestar psicosocial y el desarrollo intelectual. Así el lenguaje es el modo de representación más complejo y abstracto que se adquiere dentro de los límites de un sistema socialmente definido (Piaget, 1988).

El estudio descriptivo de los niveles del lenguaje realizado por Zeballos, Valentina; Miranda, Paloma; Rodríguez, Karina (2009); postula 4 niveles del mismo y lo que en ellos se espera; el primer nivel, morfológico está compuesto por todos los sonidos propios de cada lengua y se va desarrollando paulatinamente. En el nivel Morfosintáctico, aparecen las palabras que constituyen la unidad mínima del lenguaje. Es entre los 2 y 3 años que el menor ya posee un acceso a la asociación de dos o más palabras. El tercer Nivel; Semántico, está configurado por los significados de palabras y oraciones. De acuerdo a los precursores del lenguaje, este nivel corresponde al contenido, es decir, cómo el niño comprende y entrega respuestas con base a la organización que tiene a nivel mental de una situación determinada. El último nivel, pragmático, se refiere al uso del lenguaje; el niño aprende a utilizarlo en un contexto social a través de sonrisas, gestos y otras conductas no verbales siendo consideradas como las primeras manifestaciones de comunicación intencionadas.

Cada uno de estos niveles son fundamentales en la comunicación humana ya que, presentan funciones específicas para el procesamiento lingüístico en determinados contextos, y es en la medida que el infante crece de forma cualitativa y cuantitativa, que los niveles se desarrollan.

Las dificultades que se evidencian en el lenguaje aparecen en alguno de estos niveles de funcionamiento y es precisamente, en el perfil neuropsicológico, donde se precisa, si los niveles de 
expresión y de comprensión oral y escrita se encuentran dentro de los límites normales para la edad y capacidad intelectual del niño.

En la neuropsicología, el estudio del lenguaje tiene una larga historia; generalmente se ha dado relevancia a la patología; hay diversos estudios empíricos y propuestas de rehabilitación altamente reconocidas; sin embargo, no es habitual que se realicen estudios en poblaciones con características no patológicas. Nuestro estudio pretende contribuir en evidencia empírica en la exploración del lenguaje en población normal, el tema de los baremos es interesante cuando manejamos instrumentos neuropsicológicos, de hecho, constantemente se presentan dificultades en el momento de las valoraciones neuropsicológicas, por no poseer baremos adecuados para comparar o contrastar la ejecución de los pacientes que se evalúan en nuestro contexto cultural.

La evaluación de las habilidades lingüísticas en niños ha resultado particularmente importante en la identificación y el diagnóstico diferencial de los trastornos en el desarrollo neuropsicológico, además, los tests del lenguaje pueden proporcionar parámetros para la rehabilitación neuropsicológica y para evaluar la eficacia de los tratamientos para los trastornos del desarrollo del lenguaje.

Para determinar y evaluar el desempeño cognitivo en actividades relacionadas con el lenguaje, se aplicaron varios Test que permitieran dilucidar la estructura organizativa de los niveles del lenguaje, se aplicaron tres pruebas neuropsicológicas, Test de Vocabulario de Boston (TVB); Test de Asociación Controlada de Palabras (FAS); Token Test desarrollado por Renzi (RTT).

El estudio tuvo como objetivo describir el desempeño cognitivo de los escolares de 6 a14 años en las pruebas de lenguaje.

\section{Metodología.}

Este estudio se desarrolló bajo un paradigma de investigación empírico-analítico con un diseño descriptivo y transversal. La investigación se realizo con base al estudio de Validación de pruebas neurocognitivas, en 300 sujetos de la ciudad Medellín, realizado en el año 2009; con una muestra de 92 participantes seleccionados aleatoriamente con un 95\% de confianza y el 5\% de error, de los cuales 49 son hombres y 42 mujeres, correspondiente a estratos socioeconómicos bajo, medio y alto, con edades comprendidas entre los 6 a 14 años de la ciudad de Medellín, comparándose con los baremos del estudio antes mencionado. La administración de pruebas neuropsicológicas se realizó de forma individual.

Criterios de Inclusión: Edades 6 a 14 años, Ausencia de alteraciones neurológicas, psiquiátricas o fracaso escolar, Manifestación del consentimiento informado. Criterios de Exclusión: Trastornos en el neurodesarrollo, historia clínica con signos y síntomas de deterioro cognitivo focal o difuso, antecedentes de enfermedades del sistema nervioso central con dificultades neuropsicológicas, trastornos perceptivos visuales, auditivos y/o motores que limiten la evaluación neuropsicológica, presencia de enfermedades sistémicas agudas o crónicas no controladas que interfieran con la evaluación neuropsicológica, antecedentes o presencia de trastorno psiquiátrico mayor. 
EL Análisis estadístico se realizó en SPSS 19.0, se muestra la distribución porcentual para las variables cualitativas de tipo sociodemográfico generales y para las variables cuantitativas las medidas de tendencia central y dispersión. Además, las características demográficas se comparan mediante la prueba Chi cuadrado de homogeneidad para las variables cualitativas; y para las cuantitativas se utilizó la prueba t para la diferencia de medias en muestras independientes o en su defecto la prueba U de Mann Whitney, si la distribución no distribuye normalmente. Se evidencia el respectivo valor p y los intervalos de confianza del $95 \%$.

\section{Variables.}

- TVB: Test de Vocabulário de Boston: TVB Nominación, TVBSemántica, TVB Total.

- FAS: Test de Asociación Controlada de Palabras: F, A, S, FASTotal

- RTT: Token Test desarrollado por Renzi

\section{Instrumentos.}

Test de Vocabulário de Boston (TVB), fue desarrollado por Kaplan, Goodglass, \& Weintraub, S. (1983), consta de 16 láminas para diagnosticar afasia y 60 elementos gráficos en orden creciente de dificultad para el test de vocabulario de Boston. Evalúa afasia y otros trastornos del lenguaje: útil como guía para el tratamiento.

Test de Asociación Controlada de Palabras (FAS), desarrollado por Spreen, O. y Strauss, E. (1998). Representa una tarea de búsqueda de palabras y se asocia con trastornos de la conciencia fonológica en la lectura; es un test de fluidez verbal en el cual se le pide al paciente que diga todas las palabras que comienzan con una letra determinada. Este procedimiento es muy sensible a las disfunciones del lóbulo frontal izquierdo, particularmente a la región dorso-lateral.

Token Test desarrollado por Renzi (RTT), es una prueba específicamente de comprensión sintáctica, compuesta por material sencillo, es decir unas fichas de diferentes colores, formas y tamaños que se colocan encima de la mesa delante del paciente. La baremación de este test es clínica, no precisa adaptación y no ofrece resultados psicométricos cuantitativos. Es utilizada para evaluar pacientes con retraso mental y alteraciones del lenguaje infantil.

\section{Procedimento.}

La población fue selecionada de forma aleatória; se explico en detalle los procedimientos del estudio y se les pregunto a los participantes por historia de antecedentes neurológicos y psiquiátricos.

Posteriormente fueron citados a evaluación neuropsicológica, en ella se realizaron una serie de pruebas para medir las funciones cognitivas; con una duración de dos (2) sesiones de 50 minutos cada una. Se indagó por el consumo de medicación.

Se informo a las personas que los resultados sobre la evaluación neuropsicológica podrían proporcionarse si lo deseaban, a su médico, colegio o terapeuta, para ello debía solicitar un informe 
personal escrito, con la salvedad que a los colegios, maestros, y aseguradoras de salud no se les entregaría informes individuales de ningún participante.

\section{Resultados.}

La tabla 1 presenta las características de la muestra de estudio. La población está definida por un 53,3\% de niños y el 46,7\% de niñas; con un 51,1\% entre los 9 y 14 años de edad, la lateralidad en un 91,3\% es la derecha y pertenecientes en su gran mayoría a un estrato socioeconómico medio, es decir, estrato 3 y 4. (Ver Tabla 1)

Tabla 1. Caracterización de la población de estudio

\begin{tabular}{|c|c|c|}
\hline & Muestra & Porcentaje \\
\hline \multicolumn{3}{|l|}{ SEXO } \\
\hline Femenino & 43 & $46.7 \%$ \\
\hline Masculino & 49 & $53 . .3 \%$ \\
\hline \multicolumn{3}{|l|}{ GRUPO DE EDAD } \\
\hline 6-8 Años & 45 & $48.9 \%$ \\
\hline 9-14 Años & 47 & $51.1 \%$ \\
\hline \multicolumn{3}{|l|}{ ESCOLARIDAD } \\
\hline $1-4^{\circ}$ & 48 & $52.2 \%$ \\
\hline $5-8^{\circ}$ & 38 & $41.4 \%$ \\
\hline $9-11^{\circ}$ & 6 & $6.5 \%$ \\
\hline \multicolumn{3}{|l|}{ LATERALIDAD } \\
\hline Derecho & 84 & $91,3 \%$ \\
\hline Zurdo & 8 & $8,7 \%$ \\
\hline \multicolumn{3}{|l|}{ ESE } \\
\hline $1-3$ & 52 & $57,2 \%$ \\
\hline Fuente Propia & 39 & $42,9 \%$ \\
\hline
\end{tabular}

La tabla 2 describe las características de lenguaje por sexo; Se observa que los valores estadísticos para las variables se encuentran en puntuaciones medias para cada una, se observan diferencias en las variables para las niñas y los niños, siendo el valor promedio, en algunas mayor para los niños, diferencias que se pueden observar en la tabla 5 . A nivel general, se puede observar que el percentil 75 para variables como: TVBNominación, se encuentra en 49, siendo un valor alto, para TVBSemántico se presenta un percentil del 75 en un valor de 3, si se observa la variable TVBTotal se encuentra un promedio de 43 con una desviación estándar de 12 siendo un valor muy alto, dando cuenta de una gran dispersión para estos puntajes; para F se presentan valores todos en torno al promedio lo mismo ocurre con la variable A y con S en la variable FASTotal se presenta un promedio de 17 con una desviación de 8 siendo una desviación alta y para la variable de RTT se encuentra muy desviada del promedio, presentando valores limítrofes y elevados. (Ver Tabla 2) 
TABLA 2. Caracterización del Lenguaje por Sexo

\begin{tabular}{|c|c|c|c|c|c|c|c|}
\hline & \multicolumn{2}{|c|}{ Femenino $(n=43)$} & \multicolumn{2}{|c|}{ Masculino ( $n=49)$} & \multicolumn{2}{|c|}{ Total } & \multirow[b]{2}{*}{$\begin{array}{l}\text { Tamaño } \\
\text { del Efecto }\end{array}$} \\
\hline & Media (DS) & Rango & Media (DS) & Rango & Media (DS) & Rango & \\
\hline \multicolumn{8}{|c|}{ Test de Vocabulario de Boston } \\
\hline TVBNominación & $37 \quad(11)$ & $29-45$ & $44 \quad(11)$ & $36-51$ & $(11)$ & $32-49$ & 0.64 \\
\hline TVBSemántico & (4) & $0-4$ & $(2)$ & $0-3$ & (3) & $0 \quad-3$ & 0.33 \\
\hline TVBTotal & $40 \quad(12)$ & $30-49$ & $46 \quad(11)$ & $41-53$ & $43 \quad(12)$ & $34-52$ & 0.53 \\
\hline \multicolumn{8}{|c|}{ Test de Asociación Controlada de Palabras (FAS) } \\
\hline $\mathrm{F}$ & $5(3)$ & $3(7)$ & $5(3)$ & $3(8)$ & $5(3)$ & $3-7$ & 0.00 \\
\hline A & $6(3)$ & $4(7)$ & $6(3)$ & $4(8)$ & $6(3)$ & $4-8$ & 0.04 \\
\hline$S$ & $5(3)$ & $3(7)$ & $6(3)$ & $4(9)$ & $6(3)$ & $3-8$ & 0.34 \\
\hline FASTOTAL & $15(7)$ & $10(20)$ & $18(8)$ & $12(25)$ & $17(8)$ & $11-22$ & 0.4 \\
\hline \multicolumn{8}{|c|}{ Token Test desarrollado por Renzi } \\
\hline RTT & 138 (29) & $140(156)$ & $137(26)$ & $124(156)$ & $137(27)$ & $127-156$ & 0.04 \\
\hline
\end{tabular}

n: Frecuencia; DS: desviación Estándar.

La tabla 3 presenta las características de lenguaje por grupos de edad. Se observa que los valores estadísticos para las variables se encuentran en puntuaciones medias para cada una, se observan diferencias en las variables para las niñas y los niños, siendo el valor promedio, en algunas mayores para los niños.

Para el grupo de niños y niñas, entre 6 y 8 años, se observan puntuaciones que en general, presentan un comportamiento simétrico, aunque para las variables de TVBNominación; TVBTotal y RTT se presentan desviaciones muy altas.

Para los niños y niñas entre 9 y 14 años, se observa un comportamiento muy similar al anterior, aunque se presentan desviaciones más elevadas en las variables en las que se presentan dichas variaciones como son: TVBNominación; TVBTotal y RTT. (Ver Tabla3) 
Tabla 3. Caracterización del Lenguaje por grupos de Edad

\begin{tabular}{|c|c|c|c|c|c|c|c|}
\hline & $\begin{array}{r}\text { Entr } \\
\mathbf{a} \\
(\mathbf{n} \\
\end{array}$ & $\begin{array}{l}6 \text { y } 8 \\
\text { os } \\
45) \\
\end{array}$ & $\begin{array}{r}\text { Entre } \\
\text { a } \\
(\mathrm{n}\end{array}$ & $\begin{array}{l}\text { y } 14 \\
\text { s } \\
\text { 49) }\end{array}$ & & & \\
\hline & Medic & $(\mathrm{DE})$ & Media & $(\mathrm{DE})$ & $\mathrm{Mec}$ & $(\mathrm{DE})$ & $\begin{array}{c}\text { Tamaño } \\
\text { del } \\
\text { Efecto }\end{array}$ \\
\hline Test de Vocabula & Bosto & & & & & & \\
\hline TVBNominación & 32 & $(8)$ & 49 & $(8)$ & 42 & $(11)$ & 2.91 \\
\hline TVBSemántico & 0 & (4) & 1 & $(2)$ & 1 & (3) & 0.32 \\
\hline TVBTotal & 35 & (9) & 51 & $(8)$ & 43 & $(12)$ & 1.09 \\
\hline Test de Asociació & trolad & de Pa & bras (F & & & & \\
\hline $\mathrm{F}$ & 4 & (2) & 6 & $(3)$ & 5 & (3) & 0.79 \\
\hline A & 4 & (2) & 7 & (3) & 6 & (3) & 1.18 \\
\hline $\mathrm{S}$ & 4 & (2) & 8 & (3) & 6 & (3) & 1.57 \\
\hline FAS Total & 12 & (6) & 21 & $(7)$ & 16 & $(8)$ & 0.75 \\
\hline Token Test Desar & lo por & enzi & & & & & \\
\hline RTT & 147 & $(28)$ & 157 & $(-)$ & 148 & $(27)$ & 0.52 \\
\hline
\end{tabular}

La tabla 4 presenta la prueba t para muestras independientes por grupos de edad y lenguaje. Se puede observar, que solo existen diferencias estadísticamente significativas en cuanto al lenguaje y la variable TVBNominación con un valor de $\mathrm{p}=0,000$ y un I.C. $(-12,114 ;-18,953)$ asumiéndose varianzas iguales, con la variable TVBTotal con un valor de $\mathrm{p}=0,000$ y un I.C. $(-11,149 ;-18,584)$ asumiéndose varianzas iguales; con la variable $\mathrm{F}$ con un valor de $\mathrm{p}=0,000$ y un I.C. $(-1,245 ;-3,466)$, en la cual no se asumen varianzas iguales; con la variable A con un valor de $p=0,000$ y un I.C. $(-2,285 ;-4,394)$; con la variable $S$ con un valor de $\mathrm{p}=0,000$ y un I.C. $(-2,571 ;-4,782)$ y con la variable FASTotal con un valor de $\mathrm{p}=0,000 \mathrm{y}$ un I.C. $(-6,602 ;-11,868)$, asumiendo para estas varianzas iguales. (Ver tabla 4$)$.

Tabla 4. Prueba T para muestras independientes por grupos de Edad y
Lenguaje
\begin{tabular}{l|c|c|c|c|c}
\hline & Media & (DS) & $\mathrm{t}$ & $\mathrm{g} 1$. & Valor $\mathrm{p}$ \\
\hline Test de Vocabulario de Boston \\
\hline TVBNominación & 0,162 & $(0,688)$ & $-9,027$ & 88 & $\mathbf{P}=\mathbf{0 . 0 0 0}$ \\
\hline TVBTotal & 1,813 & $(0,182)$ & $-7,948$ & 88 & $\mathbf{P}=\mathbf{0 . 0 0 0}$ \\
\hline Test de Asociación Controlada de Palabras \\
\hline F & 4,076 & $(0,047)$ & 4,219 & 81,841 & $\mathbf{P}=\mathbf{0 . 0 0 0}$ \\
\hline A & 0,227 & $(0,635)$ & $-6,294$ & 89 & $\mathbf{P}=\mathbf{0 . 0 0 0}$ \\
\hline S & 1,748 & $(0,189)$ & $-6,609$ & 89 & $\mathbf{P}=\mathbf{0 . 0 0 0}$ \\
\hline FAS Total & 1,226 & $(0,271)$ & $-6,966$ & 89 & $\mathbf{P}=\mathbf{0 . 0 0 0}$ \\
Fuente Propia & & & &
\end{tabular}


Tabla 5 describe la prueba $t$ de la muestra independiente por sexo y lenguaje. Existen diferencias estadísticamente significativas en cuanto al lenguaje y la variable TVBNominación con un valor de $\mathrm{p}=$ 0,007 y un I.C. $(-1,807 ;-10,918)$ con la que se asumen varianzas iguales; se observan diferencias estadísticamente significativas con la variable TVBTotal con un valor de $p=0,027$ y un I.C. $(-0,627$; $10,112)$; y por último con la variable $S$ con un valor de $p=0,035$ y un I.C. $(-0,114 ;-2,724)$, asumiendo en todos los casos varianzas iguales. (Ver tabla 5).

Tabla 5. Prueba t para muestras independientes por sexo y Lenguaje

\begin{tabular}{l|l|l|l|l|l}
\hline & Media & (DS) & $\mathrm{t}$ & gl. & Valor $\mathrm{p}$ \\
\hline
\end{tabular}

Test de Vo cabulario de Boston

\begin{tabular}{l|c|c|c|c|c}
\hline TVBNominación &, 051 & $(, 823)$ & $-2,776$ & 88 &, 007 \\
\hline TVBTotal &, 268 & $(, 606)$ & $-2,250$ & 88 &, 027 \\
\hline
\end{tabular}

Test de Asociación Controlada de Palabras

\begin{tabular}{l|l|l|l|l|l}
\hline $\mathrm{S}$ & 1,138 & $(, 289)$ & $-2,139$ & 89 &, 035 \\
Fuente Propia & & & & &
\end{tabular}

\section{Discusión y conclusiones.}

El principal objetivo de este estudio fue el de suministrar una descripción del desempeño cognitivo en pruebas de lenguaje en niños de 6 a 14 años; al llevarse a cabo el estudio en una población normal permite establecer baremos en nuestro contexto; tema bien importante en América Latina, debido a que constantemente se presentan dificultades en el momento de las valoraciones neuropsicológicas, por no poseer baremos adecuados para comparar o contrastar la ejecución de los pacientes que se evalúan. Precisamente los resultados de esta investigación permiten obtener una base de datos normativos para los instrumentos más utilizados en la evaluación del lenguaje, de tal manera que se pueden hacer comparaciones pertinentes entre diferentes muestras, con el fin de determinar si existen o no diferencias significativas entre las mismas, y realizar valoraciones neuropsicológicas más veraces y eficaces.

En nuestra investigación el factor edad influenció en la realización de los sujetos en los tres tests. En el test de vocabulario de Boston, el grupo de niños de 9 a 14 años les fue significativamente mejor que a los niños de 6 a 8 años. En la FAS y en el token test se hallan del mismo modo, diferencias por edad. No se comprobó una diferencia significativa entre los géneros en cuanto a la realización de los tests evaluados. Estos resultados concuerdan con los de Riva (2000).

Se halla que la apropiación y uso del lenguaje es un mediador en el desarrollo cognitivo y del curso de los procesos mentales. De tal forma, que lo establecido permite afirmar que el rol central del lenguaje, 
lleva consigo el manejo conceptual y reflexivo de los procesos ejecutivos en los niños. Estos resultados están de acuerdo con los resultados previos en otros países en los que quedó demostrada una clara mejoría en la realización de acuerdo con la edad en los tests de fluidez verbal y en el test de denominación de Boston. Caso de investigaciones como la de Bentes (2007) en Brasil que arrojaron por medio de su estudio que no hay diferencias entre la realización de los niños y niñas en ninguno de los tests, pero la edad influyó en la realización de los sujetos en los tres tests.

En los estudios y en la elaboración de pruebas neuropsicológicas se distinguen los grupos de edades. Se halla que las habilidades intelectuales varían a través del tiempo y así mismo los puntajes en pruebas de evaluación neuropsicológica. Las conclusiones de los trabajos empíricos coinciden en que la forma en que una madre se dirige a un niño es más simple, redundante y gramatical que cuando los adultos hablan con otro adulto. A medida que mejora la competencia infantil, la adaptación adulta va aproximando su acción a los niveles propios del habla entre adultos (R.A. Clemente, L. Villanueva, 1999).

No es usual que las tablas normativas para pruebas de evaluación neuropsicológica presenten normas separadas para hombres y mujeres. Este efecto del sexo sobre las habilidades intelectuales, en particular, las habilidades lingüísticas, ha sido cuestionado (Wallentin, 2009), sugiriéndose que no existen datos suficientemente sólidos que lo respalden lo que apoya los resultados de la presente investigación. En todos los estudios normativos adelantados, (1) no se ha encontrado que haya ninguna diferencia significativa en ninguna prueba neuropsicológica entre hombres y mujeres si se toman sujetos con 12 o más años de educación; (2) nunca se ha encontrado en sujetos con menos de 12 años de educación que las mujeres aventajen a los hombres en ninguna prueba (Ardila, 2012). Por ello, diversos estudios muestran que varía la comunicación según el nivel de desarrollo y no según el sexo, evidenciando que las mujeres no se desempeñan mejor que los hombres en pruebas de lenguaje; no existen diferencias neuropsicológicas significativas en pruebas neuropsicológicas por sexo.

Los resultados conseguidos hasta el momento deben considerarse preliminares. Se necesitan llevar a cabo estudios con muestras mayores, diferentes niveles socioeconómicos y otros grupos de edad. Los estudios con poblaciones clínicas son particularmente importantes para acumular evidencias de validez discriminativa. Sin embargo, los resultados aquí presentados pueden ser útiles en la elección de dificultades de lenguaje en niños con características de edad y sociales similares. 


\section{Referencias Biblibográfica.}

Alvarado, S. \& Suarez, M. (2009). Las transiciones escolares: una oportunidad de desarrollo integral para niños y niñas. Revista Latinoamericana de Ciencias Sociales, Niñez y Juventud. Vol. 7., pàg. 907-928. (1997). Desarrollo Humano. Medellín. CINDE.

Arboleda, A., Lopera, J., Hincapié, L., Giraldo, M., Pineda, D., Lopera, F. \& Lopera, E. (2007). Trastorno específico del desarrollo del lenguaje: problema selectivo o generalizado de la cognición. Revista de Neurología. Vol. 44 (10). Pág. 596-600.

Ygual, A., Cervera, J., \& Rosso, P. (2008). Utilidad del análisis fonológico en la terapia del lenguaje. Revista de neurología. Pág. 97-100.

Campillo, D.,\& Garcia, E. (2005). Origen y evolución del lenguaje. Revista de Neurología. Pág. 5-10.

D. del Rio, M., Santiuste, A. Capilla, F. Maestú, P., Campo, A. \& Fernández, T. (2005). Bases neurológicas del lenguaje. Aportaciones desde la magnetoencefalografia. Revista de Neurología. Pág. 109-114.

Fernandes, L., Bentes, RC., Figueiredo, P., Brandão, D., Costa, S., Parizzi, A., Borges, W., \& Salgado, J. (2007). Normalización de una batería de tests para evaluar las habilidades de comprensión del lenguaje, fluidez verbal y denominación en niños brasileños de 7 a 10 años: resultados preliminares. Revista de Neurología .Vol. 44., pág. 275-280.

Agudelo, G., Cuentos, F., Domezáin, M. \& Pascual, B. (2006). Repetición de pseudopalabras en niños españoles con trastorno específico del lenguaje: Marcador psicolinguistico. Revista de neurología. Pág. 201-208.

Galarza, J., Padilla L. \& Bonilla, J. (2005). Evaluación neuropsicológica de una muestra de niños de 5 a 12 años con instrucción escolar bilingüe. Interação em Psicologia, Vol. 9 (1), pág. 125-130 1. Universidad Autónoma de Baja California, México.

Hincapié, L., Giraldo, M., Castro, R., Lopera, F., Pineda, D. \& Lopera, E. (2007). Propiedades lingüísticas de los trastornos específicos del desarrollo del lenguaje. Revista Latinoamericana de Psicología. Vol.39., Nº1.

Noé,E., Irimia, P., Matínez, E. \& Luquin, MR. (2000). Estudio neuropsicológico y de perfusión cerebral mediante spect con 99mtc-hmpao en pacientes con enfermedad de parkinson "de novo". Departamento de neurología. Clínica universitaria de navarra. Pamplona. España. I Congreso Virtual de Psiquiatria 1 de Febrero - 15 de Marzo; Conferencia 19-CI-D: [62 pantallas]. Disponible en: http://www.psiquiatria.com/articulos/para_revisar/2047/.

Clemente, R. \& Villanueva, L. (1999). El desarrollo del lenguaje: los prerrequisitos psicosociales de la comunicación. Revista de Neurología. Pág. 100-105. 
Monfort, M. (2004). Intervención en niños con trastornos pragmáticos del lenguaje y la comunicación. Revista de Neurología. Pág. 85-87.

Puyuelo, M. (2001). Psicología, audición y lenguaje en diferentes cuadros infantiles. Aspectos comunicativos y neuropsicológicos. Revista de Neurología. Pág. 975-980.

Piaget, J. \& Inhelder, B. (1984). Psicología del Niño. Madrid, España. Editorial: Morata.

Pinel, J. (2000). Biopsicología. Madrid, España. Ediciones: Prentice-Hall.

Puente, A. (1989). Psicología Cognoscitiva, Desarrollo y Perspectivas. Caracas, Venezuela. Ediciones: Mc Graw-Hill.

Rodríguez, M., Zapata. M. \& Puentes, P. (2008). Perfil neuropsicológico de escolares con trastornos específicos del aprendizaje de instituciones educativas de Barranquilla, Colombia. Acta Neurol Colomb Vol. 24 No. 2.

Rodríguez ,W. (2011). Aprendizaje, desarrollo y evaluación en contextos escolares: consideraciones teóricas y prácticas desde el enfoque. Histórico cultural. Revista Electrónica Actualidades Investigativas en Educación, vol. 11, núm. 1, pág. 1-36. Universidad de Costa Rica, San Pedro de Montes de Oca, Costa Rica.

Rosselli, M., Matute, E., Ardila, A., Botero, V., Tangarife, G., Echeverría, S., Arbelaez, C., Mejía, M., Méndez, L., Villa, P. \& Ocampo, P. (2004). Evaluación Neuropsicológica Infantil (ENI): una batería para la evaluación de niños entre 5 y 16 años de edad. Estudio normativo colombiano. Revista de Neurología. Vol. 38 (8). Pág. 720-731.

Fernández, TTejero, C., Santos, S., Pérez,C., Piñol, G., Mostacero, E. \& Pascual, L. (2006) Lenguaje y deterioro cognitivo: un estudio semiológico en denominación visual. Revista de Neurología. Pág. 578-583.

Vigotsky, L. (1978). El Desarrollo de los Procesos Psicológicos Superiores. Barcelona, España. Ediciones: Crítica. 